\title{
Managing Quality of Service with Soft Constraints
}

\author{
Francesco Santini \\ IMT School of Advanced Studies \\ Piazza San Ponziano 6, Lucca, Italy \\ http://santini.francesco.googlepages.com \\ e-mail: f.santini@imtlucca.it
}

\section{Introduction}

The term "quality" as it is commonly understood in the context of Quality of Service (QoS) is "something" by which a user of the service (in a very large meaning) will judge how good the service is. In this research project we mainly focus our attention to three areas related with QoS: i) Networks, ii) Web Services and iii) Trust Management (TM).

As defined in (Crawley et al. 1998), QoS is "a set of service requirements to be met by the network while transporting a flow", where a flow is "a packet stream from source to a destination (unicast or multicast) with an associated Quality of Service (QoS)". To be implemented and subsequently satisfied, network requirements have to be expressed in some measurable QoS metrics: well-known metrics include bandwidth, hops, delay, jitter, cost and loss probability. With Constraint-Based Routing (CBR) (Younis and Fahmy 2003) we refer to a class of routing algorithms that base path selection decisions on a set of requirements or constraints, in addition to the destination. The main objectives are to meet the QoS requirements of applications and to optimize the global network resource usage.

Web Services (WS) are maturing as a technology that allows for the integration of applications belonging to different administrative domains, enabling much faster and efficient business-to-business arrangements. A Service Level Agreement (SLA) is an agreement regarding the guarantees of a WS: it defines mutual understandings and expectations of a service between the service provider and service consumers, and clearly involves many QoS indications and requests about the traded service (Lee et al. 2003).

Trust and Reputation Systems represent a significant trend in decision support for Internet mediated service provision (Jøsang, Ismail, and Boyd 2006). The basic idea is to let parties rate each other, for example after the completion of a transaction, and use the aggregated ratings about a given party to derive a trust or reputation score, which can assist other parties in deciding whether or not to transact with that party in the future. Trust describes how much the reliability in the service is rated, and therefore we can easily consider it as a QoS feature.

The aim of my $\mathrm{PhD}$ thesis is to provide expressive means (e.g. languages) in order to model and solve these frame-

Copyright (c) 2008, Association for the Advancement of Artificial Intelligence (www.aaai.org). All rights reserved. works with the help of Soft Constraints (Bistarelli 2004), benefiting from Artificial Intelligence background to tackle this kind of optimization problems. Soft constraints will represent the needs of the parties on the traded resources and the consistency value of the store represents a feedback on the current agreement. Using soft constraints gives to the service provider and the clients more flexibility in expressing their requests w.r.t. crisp constraints, and therefore there are more chances to reach a shared agreement. Moreover, the cost model is very adaptable to the specific problem, since it is parametric with the chosen semiring (Bistarelli 2004).

\section{Past Works}

We suggested a formal model to represent and solve the multicast routing problem in multicast networks with QoS requirements (e.g. bandwidth and delay) (Bistarelli et al. 2007; Bistarelli and Santini 2008a). In this model we describe how to represent a network configuration in its corresponding and-or graph, mapping network nodes to andor graph nodes and links to graph connectors. Afterwards, we propose the Soft Constraint Logic Programming (SCLP) (Bistarelli and Rossi 2001) framework as a convenient declarative programming environment in which to specify and solve such problem. In particular, we show how to represent an and-or graph as an SCLP program, and how the semantics of such a program computes the best tree in the corresponding weighted and-or graph. This best tree can be used to shape the optimized multicast tree that ensures QoS requirements on the corresponding network. Qos features can be represented with c-semirings algebraic structures.

We suggested the new concept of multitrust (Bistarelli and Santini 2008b; 2008c): multitrust extends the usual trust relationship from couples of individuals to one trustor and multiple trustees in a correlated way. The correlation can be expressed in terms of time (i.e. at the same time), modalities (i.e. with the same behavior) or collaboration among the trustees. Some everyday examples can be found when downloading a file from multiple sources in peer-to-peer networks, or, in general, when a task must/can be accomplished with the help of many individuals acting together and a trust feedback must be found for the whole process. We propose SCLP as a mean to quickly represent and evaluate trust propagation for this scenario.

Moreover, we extended the Datalog language (we call 
it Datalog $^{W}$ ) in order to deal with weights on ground facts and to consequently compute a feedback result for the goal satisfaction (Bistarelli, Martinelli, and Santini 2008b; 2008a). The weights are chosen from a proper c-semiring. As a second step, we use Datalog ${ }^{W}$ as the basis to give a uniform semantics to declarative $R T^{W}$ (TM) language family, in order to represent trust levels based on c-semirings. In this way it is possible to manage a score corresponding to a preference or cost associated to the revealed credentials, instead of a plain "yes or no" authorization result. The approach is rather generic and could be applied to other trust management languages based on Datalog, as a semantic sublayer to represent trust management languages where the trust level is relevant.

\section{Current and Future Works}

In order to enrich our SCLP framework for QoS routing, we would like to investigate how c-semirings can be used inside ad-hoc algorithms to find Minimum Spanning Trees and Steiner Trees structures over a network (for multicast).

In addition, we plan to extend the Soft Concurrent Constraint (SCC) (Bistarelli, Montanari, and Rossi 2006) language to allow the non-monotonic evolution of the constraint store (Bistarelli and Santini 2007). The novelty mainly consists in the possibility of removing soft constraints from the store and to consequently deal with open and reactive systems. To accomplish this, we will introduce some new operations (e.g. a retract(c), where $c$ is the constraint to remove). We present this framework as a possible solution to the management of resources (e.g. web services and network resource allocation) that need a given Quality of Service, which for us is related to all the possible non-functional characteristics associated to the resource, e.g. availability, interoperability and execution time.

Our intention is also to further extend the SCC language in order to join together the expressive capabilities of soft constraints and timing mechanisms (Bistarelli et al. 2008). The agents modeled with this language will be able to deal with time and preference dependent decisions that can often be found during complex interactions as, for example, auctions: the cost for a service or a good can be raised or lowered during the auction process. Mechanisms as timeout and interrupt can be very useful when waiting for pending conditions or when triggering some new necessary actions. From the point of view of the service provider, time awareness can be used at expiry time to force the release of the resources dedicated to a client, or to alert the client if new resources are now available.

Moreover, we are currently investigating the feasibility of a distributed soft constraint store, where variables and constraints are distributed among all the agents, and thus the knowledge of the problem is not concentrated in a single point only. This requirement is common in many practical application, and surely for (SLA) negotiating entities.

We want to investigate the extension of TM languages with soft constraints, for example by finding which classes of soft constraints are tractable from the computational point of view. This research line could affect also other important fields, as Soft Constraint Databases. We will also develop a tool in Constraint Handling Rules to practically solve the "Datalog with soft constraints" problem.

At last, we plan to model the composition of trust networks and their analysis from a security point of view, e.g. by adding/removing a link or by increasing/reducing the associated trust score.

\section{References}

Bistarelli, S., and Rossi, F. 2001. Semiring-based constraint logic programming: syntax and semantics. $A C M$ Trans. Program. Lang. Syst. 23(1):1-29.

Bistarelli, S., and Santini, F. 2007. Designing a nonmonotonic soft concurrent constraint language for SLA management. Technical Report TR-18/2007, IIT - CNR.

Bistarelli, S., and Santini, F. 2008a. A formal and practical framework for constraint-based routing. In To appear in International Conference on Networking (ICN '08). IEEE.

Bistarelli, S., and Santini, F. 2008b. Propagating multitrust within trust networks. In To appear in "TRECK”, Symposium on Applied Computing '08. ACM.

Bistarelli, S., and Santini, F. 2008c. SCLP for trust propagation in small-world networks. In To appear in Recent Advances in Constraints '08. LNAI.

Bistarelli, S.; Montanari, U.; Rossi, F.; and Santini, F. 2007. Modelling multicast QoS routing by using best-tree search in and-or graphs and soft constraint logic programming. Electr. Notes Theor. Comput. Sci. 190(3):111-127.

Bistarelli, S.; Gabrielli, M.; Meo, M. C.; and Santini, F. 2008. Timed concurrent constraint programs. In To appear in COORDINATION'08. Springer.

Bistarelli, S.; Martinelli, F.; and Santini, F. 2008a. A semantic foundation for trust management languages with weights: An application to the RT family. In To appear in Autonomic and Trusted Computing (ATC '08). Springer.

Bistarelli, S.; Martinelli, F.; and Santini, F. 2008 b. Weighted datalog and levels of trust. In To appear in Advances in Policy Enforcement (APE) colocated with ARES. IEEE.

Bistarelli, S.; Montanari, U.; and Rossi, F. 2006. Soft concurrent constraint programming. ACM Trans. Comput. Logic 7(3):563-589.

Bistarelli, S. 2004. Semirings for Soft Constraint Solving and Programming, volume 2962 of LNCS. Springer.

Crawley, E.; Nair, R.; Rajagopalan, B.; and Sandick, H. 1998. RFC 2386: A framework for QoS-based routing in the Internet. Informational.

Jøsang, A.; Ismail, R.; and Boyd, C. 2006. A survey of trust and reputation systems for online service provision. To appear in Decision Support Systems.

Lee, K.; J. Jeon, W. L.; Jeong, S.-H.; and Park, S. W. 2003. QoS for web services: Requirements and possible approaches. W3C Note, available at http://www.w3c.or.kr/kroffice/TR/2003/ws-qos/.

Younis, O., and Fahmy, S. 2003. Constraint-based routing in the internet: Basic principles and recent research. IEEE Communications Surveys and Tutorials 5(1):2-13. 